Wickens M. The 3 '-untranslated regions of c-mos and cyclin mRNAs stimulate translation by regulating cytoplasmic polyadenylation. Genes Dev. 1994;8(8):926-938.

6. Mays-Hoopes LL, Bolen J, Riggs AD, Singer-Sam J. Preparation of spermatogonia, spermatocytes, and round spermatids for analysis of gene expression using fluorescence-activated cell sorting. Biol Reprod. 1995;53(5):1003-1011.

7. Giorgini F, Davies HG, Braun RE. Translational repression by MSY4 inhibits spermatid differentiation in mice. Development. 2002;129(15):3669-3679.

8. Yang J, Morales CR, Medvedev S, Schultz RM, Hecht NB. In the absence of the mouse DNA/RNA-binding protein MSY2, messenger RNA instability leads to spermatogenic arrest. Biol Reprod. 2007;76(1):48-54.

9. Kleene KC. Poly(A) shortening accompanies the activation of translation of five mRNAs dur- ing spermiogenesis in the mouse. Development. 1989;106(2):367-373.

10. Yanagiya A, Delbes G, Svitkin YV, Robaire B, Sonenberg N. The poly(A)-binding protein partner Paip2a controls translation during late spermiogenesis in mice. J Clin Invest. 2010;120(9):3389-3400.

11. Derry MC, Yanagiya A, Martineau Y, Sonenberg N. Regulation of poly(A)-binding protein through PABP-interacting proteins. Cold Spring Harb Symp Ouant Biol. 2006;71:537-543.

12. Craig AW, Haghighat A, Yu AT, Sonenberg N. Interaction of polyadenylate-binding protein with the eIF4G homologue PAIP enhances translation. Nature. 1998;392(6675):520-523.

13. Martineau $\mathrm{Y}$, et al. Poly(A)-binding protein-interacting protein 1 binds to eukaryotic translation initiation factor 3 to stimulate translation. Mol Cell Biol. 2008;28(21):6658-6667.
14. Khaleghpour K, et al. Translational repression by a novel partner of human poly(A) binding protein, Paip2. Mol Cell. 2001;7(1):205-216.

15. Berlanga JJ, Baass A, Sonenberg N. Regulation of poly(A) binding protein function in translation: Characterization of the Paip2 homolog, Paip2B. RNA. 2006;12(8):1556-1568.

16. Yoshida M, et al. Poly(A) binding protein (PABP) homeostasis is mediated by the stability of its inhibitor, Paip2. EMBO J. 2006;25(9):1934-1944.

17. Wu J, Bag J. Negative control of the poly(A)-binding protein mRNA translation is mediated by the adenine-rich region of its $5^{\prime}$-untranslated region. J Biol Chem. 1998;273(51):34535-34542.

18. Radford HE, Meijer HA, de Moor CH. Translational control by cytoplasmic polyadenylation in Xenopus oocytes. Biochim Biophys Acta. 2008;1779(4):217-229.

\title{
Transgenic animals may help resolve a sticky situation in cystic fibrosis
}

\author{
Jonathan $\mathrm{H}$. Widdicombe
}

Department of Physiology and Membrane Biology, University of California Davis, Davis, California, USA.

\begin{abstract}
Cystic fibrosis (CF) is caused by defects in the CFTR, a cAMP-activated $\mathrm{Cl}^{-}$ channel of epithelia. The resulting reduction in epithelial fluid transport creates abnormally viscous secretions from airway mucous glands that may be a major factor in CF pathology. Mouse airways have few mucous glands, and the mouse model of $\mathrm{CF}$ exhibits no significant airway disease. Pigs and ferrets, however, have approximately the same number of airway mucous glands as humans. In this issue of the JCI, three independent research groups conclude that changes in airway mucous gland function in CFTR-deficient animals of these species resemble the changes seen in human CF. It is expected, therefore, that these animals will develop lung disease similar to human CF and prove to be valuable models on which to test potential therapies.
\end{abstract}

The body's main defense against large inhaled particles is the "mucociliary clearance system." This system traps particles in a blanket of mucus and then moves the mucus and ensnared particles out of the airways using cilia in the apical membrane of the surface epithelium (1). In the larger airways, the great majority of mucus comes from submucosal glands (2), and mucous secretions from these glands are abnormally viscous in individuals with cystic fibrosis (CF) (3). It is believed that this causes the mucus to be poorly cleared by cilia and it accumulates and provides a home for inhaled microorganisms. An inflammatory cascade ensues that clogs the airways with mucous secretions, bacteria, leukocytes,

Conflict of interest: The author has declared that no conflict of interest exists.

Citation for this article: J Clin Invest. 2010; 120(9):3093-3096. doi:10.1172/JCI44235. and plasma transudate. By restoring normal viscosity to $\mathrm{CF}$ airway mucous gland secretions, it is to be hoped that much of this pathology can be prevented.

Mouse airways contain few mucous glands, and the mouse model of CF shows little airway pathology (4). However, more direct analyses of the role of mucous glands in the airway pathology of CF are now possible, given the recent development of CFTR-deficient pigs and ferrets $(5,6)$; both species have good numbers of airway glands. In this issue of the $J C I$, three independent research groups report on their efforts to understand airway gland function in these new animal models of CF (7-9).

\section{Airway mucous gland secretion in CF}

Water accounts for approximately $98 \%$ of airway gland mucous secretions, and water flow into the gland lumen is driven by local osmotic gradients generated by active $\mathrm{Cl}^{-}$secretion. This $\mathrm{Cl}^{-}$secretion requires simultaneous activity of $\mathrm{Cl}^{-}$channels (either cAMP or $\mathrm{Ca}^{2+}$ activated) in the apical membrane and $\mathrm{K}^{+}$channels in the basolateral membrane. In CF secretory responses of airway glands to agents that elevate cAMP are almost completely lost, as the only cAMP-activated $\mathrm{Cl}^{-}$channel in the apical membrane is CFTR (10). Secretory responses to agents, such as substance $\mathrm{P}$, that moderately elevate intracellular $\mathrm{Ca}^{2+}$ concentration $\left(\left[\mathrm{Ca}^{2+}\right]_{i}\right)$ are also quite markedly reduced in CF (11). This is because $\left[\mathrm{Ca}^{2+}\right]_{i}$ is not elevated sufficiently to have much effect on $\mathrm{Ca}^{2+}$-activated $\mathrm{Cl}^{-}$ channels (CaCCs) in the apical membrane, but $\mathrm{Ca}^{2+}$-dependent basolateral $\mathrm{K}^{+}$channels are opened, thereby hyperpolarizing the apical membrane and increasing the driving force for $\mathrm{Cl}^{-}$exit through constitutively open CFTR. By contrast, responses to cholinergic agents are less affected in CF, because these agents have a larger effect on $\left[\mathrm{Ca}^{2+}\right]_{i}$ and cause substantial activation of $\mathrm{CaCCs}(10-12)$. Finally, in nonCF airway glands, subthreshold doses of cAMP-elevating and $\mathrm{Ca}^{2+}$-elevating agents show synergy; neither alone stimulates gland fluid secretion, but they do so in combination, because one opens CFTR and the other opens basolateral $\mathrm{Ca}^{2+}$-activated $\mathrm{K}^{+}$channels $(10-12)$. This synergy is lost in CF (10). 
Table 1

Properties of tracheal glands in humans, ferrets, and pigs

\begin{tabular}{lcccc} 
Species & $\begin{array}{c}\text { Gland openings } \\
\left(\mathbf{p e r ~} \mathbf{~ m}^{2}\right)\end{array}$ & $\begin{array}{c}\text { Gland volume } \\
\left(\mu \mathrm{l} / \mathbf{c m}^{2}\right)\end{array}$ & $\begin{array}{c}\text { Maximal flow } \\
\left(\mathbf{n l ~ m i n} \mathbf{m}^{-1} \times \text { gland }^{-1}\right)\end{array}$ & Refs. \\
Human & $\sim 1$ & $15,16^{\mathrm{A}}, 20^{\mathrm{B}}$ & $\sim 3^{\mathrm{C}}$ & $10,13,16,21$ \\
Pig & $0.6^{\mathrm{A}}, 1.3^{\mathrm{A}}, 1.2^{\mathrm{B}}$ & $8^{\mathrm{A}}, 13^{\mathrm{B}}$ & $\sim 12^{\mathrm{D}}, \sim 4^{\mathrm{C}}$ & 12,21 \\
Ferret & $\sim 1.5^{\mathrm{A}}, 1.8^{\mathrm{B}}$ & 0.6 & $\sim 0.4$ & 19,22 \\
Ferret xenograft & $\sim 2$ & $?$ & 0.06 & 8 \\
\hline
\end{tabular}

${ }^{A}$ Anterior cartilaginous portion or ${ }^{B}$ posterior membranous portion of the trachea. ${ }^{C}$ Sustained or Dtransient flows.

\section{Airway mucous gland secretion in the CFTR-deficient pig}

There were several reasons for believing that a CFTR-deficient pig would prove an excellent model of altered gland function in CF. Numbers, size, and maximal flow rates of glands in pig and human tracheas are very similar (Table 1 ). So too is the specific potency of gland secretagogues: cholinergic agents, such as carbachol or methacholine, elevate $\left[\mathrm{Ca}^{2+}\right]_{\mathrm{i}}$ and are several times more effective at inducing secretion than cAMP-elevating agents, such as forskolin or vasoactive intestinal peptide (VIP); $\alpha$-adrenergic agents, such as phenylephrine, are least effective; and substance $\mathrm{P}$ occupies an intermediate position (10-12). Also, in tracheas of different species and in different generations of human airways, glands disappear as airway diameters become less than about $2 \mathrm{~mm}(13,14)$. Therefore, given that pig and human lungs are similar in size, glands should show a similar distribution along the airways of the two species.

The CFTR-deficient transgenic pig was generated two years ago (6), and the paper by Joo et al. (7) in this issue of the JCI provides the first comparison of airway gland mucus secretion in CF and normal piglets. Piglets had to be used, as CF pigs die of gut problems shortly after birth. To measure gland secretion, the mucosal surface of a piece of airway wall was coated with paraffin oil, and gland secretions were visualized as spherical droplets forming under the oil. The results were gratifying - CF pig glands behaved very much like human CF glands. The secretory response to forskolin was lost as was the synergy between forskolin and carbachol. The secretory responses to substance $\mathrm{P}$ were reduced by approximately $75 \%$. The secretory responses to carbachol were reduced by approximately $40 \%$, a finding largely ascribable to a reduction in gland size in CF piglets. It is not known in human CF whether airway glands are abnormally small at birth, but this should definitely be investigated. Of course, with chronic disease they become markedly hypertrophied (15).

\section{Airway mucous gland secretion in the CFTR-deficient ferret}

The paper by Sun et al. (8) describes the overall pathology of the CF ferret. $\mathrm{CFTR}^{+/+}$, $\mathrm{CFTR}^{+-}$, and $\mathrm{CFTR}^{-/-}$animals were born in the expected Mendelian ratio of 1:2:1, indicating no fetal mortality. However, all CFTR $^{-/-}$kits died within 4 days of birth, either from meconium ileus or from poor nutrient absorption in the gut. Histological lesions were present in the pancreas, and vasa deferentia were absent or degenerate. There was histological evidence of pneumonia, and blood chemistry was consistent with liver disease.

In humans (and presumably pigs), all airway glands form long before birth
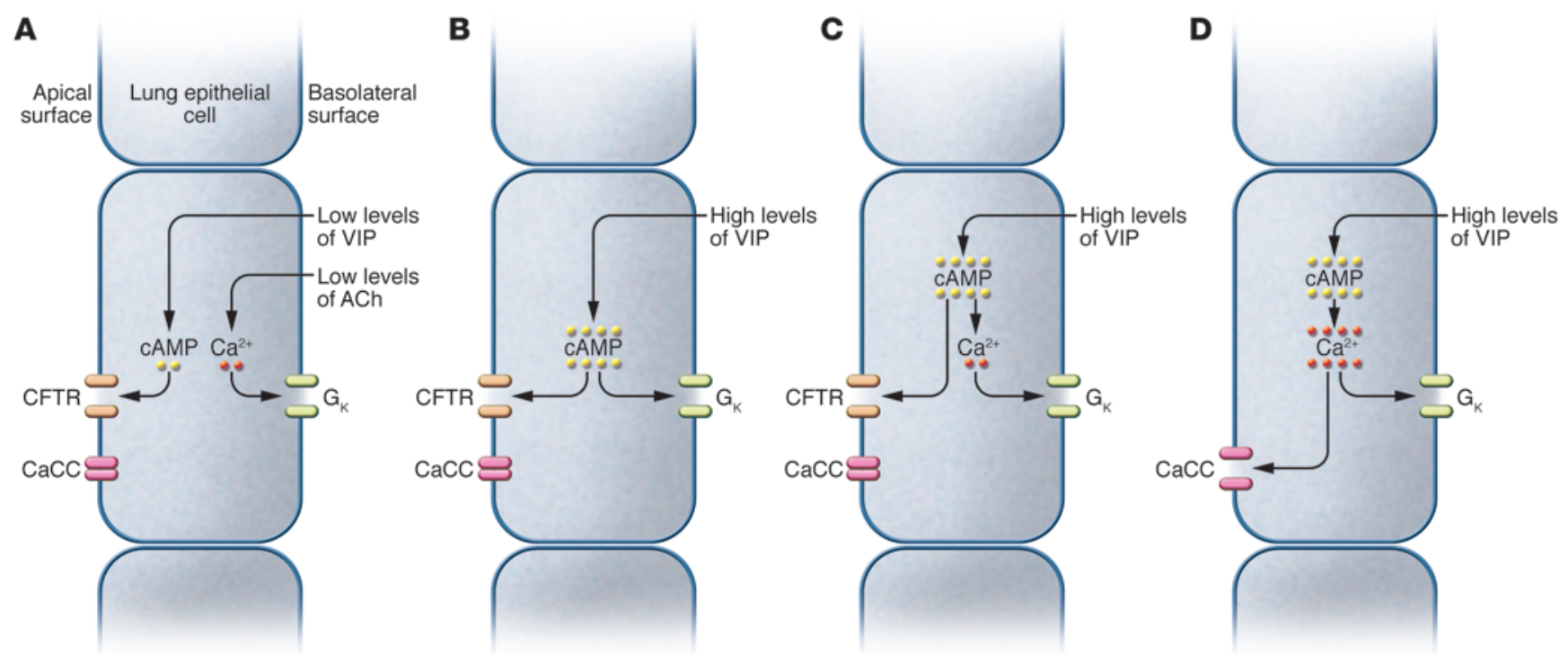

Figure 1

Means by which VIP (or other cAMP-elevating agents) stimulates $\mathrm{Cl}^{-}$secretion by airway gland serous cells. (A) Low levels of VIP are insufficient to stimulate $\mathrm{Cl}^{-}$secretion but do elevate cAMP sufficiently to open CFTR. Low levels of acetylcholine (ACh) (also subthreshold for secretion) raise $\left[\mathrm{Ca}^{2+}\right]_{i}$ sufficiently to open basolateral $\mathrm{K}^{+}$channels $\left(\mathrm{G}_{\mathrm{K}}\right)$. Together, the two agents induce $\mathrm{Cl}^{-}$secretion, as previously suggested by $\mathrm{Choi}$ et al. (10). (B) High levels of VIP elevate cAMP sufficiently to activate both $\mathrm{Cl}^{-}$and $\mathrm{K}^{+}$channels, as previously suggested by Choi et al. (10). (C) cAMP elevates $\left[\mathrm{Ca}^{2+}\right]_{\text {, }}$ which then activates basolateral $\mathrm{K}^{+}$channels, as described by Lee and Foskett (9). (D) If the effects of cAMP on [Ca $\left.{ }^{2+}\right]_{i}$ are somehow enhanced then VIP can induce $\mathrm{Cl}^{-}$secretion in CF cells by activating CaCC, as suggested by Lee and Foskett (9). 
(16). In the ferret, by contrast, airway glands form predominantly after birth (17). This, coupled with the neonatal mortality of CF ferrets, caused Sun et al. to resort to the "tracheal xenograft model" to study gland function. Using this approach, tracheas from newly born ferrets were implanted under the skin of immune-deficient mice. After five weeks, differentiated glands had formed. The xenografts were then removed from the mice, and gland secretions were visualized as secretory droplets under oil. As in pigs and humans, the secretory response to forskolin was essentially abolished in the airway glands from CF animals. The response of $\mathrm{CF}$ ferrets to methacholine was also reduced (by approximately 50\%), as it is in human nasal glands (18).

An important contribution of the study by Sun et al. (8) was the prevention of the neonatal mortality in CF ferrets with a combination of oral ursodeoxycholic acid (to aid fat emulsification) and an oral proton-pump inhibitor (to compensate for the lack of alkaline pancreatic secretions). However, even if CF ferrets achieve adulthood, they may prove less useful than adult CF pigs for studies of how altered airway gland function contributes to airway pathology in CF. Thus, not only do ferret glands develop differently from those in the human, but they are also appreciably smaller than human glands, with lower flow rates (Table 1). There are also differences in the neurohumoral regulation of airway gland secretion between ferrets and humans. In particular, the ferret, like the other carnivore studied, the cat, has airway glands that show large secretory responses to the $\alpha$-adrenergic agent, phenylephrine $(19,20)$; responses of pig and human glands to this agent are negligible (20).

\section{Interaction between cAMP and $\mathrm{Ca}^{2+}$ in airway gland cells}

The paper by Lee and Foskett (9) concerns the regulation of $\mathrm{Cl}^{-}$secretion in serous cells. These cells line the acini of airway glands and are believed to be the primary source of the liquid component of gland secretions. The authors studied secretory responses of isolated serous acini from pigs and humans, both with and without CF. Simultaneous opening of apical $\mathrm{Cl}^{-}$channels and basolateral $\mathrm{K}^{+}$channels, in response to secretagogues, resulted in $\mathrm{KCl}$ loss and cellular shrinkage, measured by Lee and Foskett using differential inter- ference microscopy. Chloride permeability and $\left[\mathrm{Ca}^{2+}\right]_{\mathrm{i}}$ were measured using fluorescent probes. As expected, the secretory response of non-CF acini to elevation of cAMP was CFTR mediated. Surprisingly, however, the response depended on CAMPmediated elevation of $\left[\mathrm{Ca}^{2+}\right]_{\mathrm{i}}$.

Choi et al. (10) had previously proposed that airway gland serous cells show two types of $\mathrm{Cl}^{-}$(and water) secretion. In the "emergency response," high levels of acetylcholine activate apical membrane $\mathrm{CaCCs}$ and promote fluid flows that are not much altered in CF. By contrast, a low baseline secretion of $\mathrm{Cl}^{-}$is maintained by a synergy between two neurotransmitters: VIP, which raises CAMP and opens CFTR, and low levels of acetylcholine, which elevate $\left[\mathrm{Ca}^{2+}\right]_{\mathrm{i}}$ sufficiently to open basolateral $\mathrm{K}^{+}$channels but not apical membrane CaCCs (Figure 1A). This baseline secretion is absent in CF. They further proposed that high concentrations of VIP can act without acetylcholine on non-CF glands, because cAMP levels become high enough to open cAMP-activated $\mathrm{K}^{+}$channels (10) (Figure 1B). Lee and Foskett, however, found that increases in CAMP (induced by either VIP or forskolin) led to increases in $\left[\mathrm{Ca}^{2+}\right]_{i}$, and this, not cAMP, was responsible for activation of the basolateral $\mathrm{K}^{+}$conductance (9) (Figure 1C). The importance of this finding is that pharmacological enhancement of the link between cAMP and $\left[\mathrm{Ca}^{2+}\right]_{i}$ (or $\left[\mathrm{Ca}^{2+}\right]_{i}$ and $\mathrm{CaCCs}$ ) would allow cAMP-elevating mediators such as VIP to stimulate $\mathrm{Cl}^{-}$ secretion in CF cells by activating $\mathrm{CaCCs}$ (Figure 1D). The authors also showed that high levels of intracellular cAMP (induced using either VIP or forskolin) enhanced the effects of acetylcholine on $\left[\mathrm{Ca}^{2+}\right]_{\mathrm{i}}(9)$. Thus, cAMP-elevating agents, such as phosphodiesterase inhibitors, could potentiate the effects of low-level cholinergic stimulation on CFTR-independent $\mathrm{Cl}^{-}$secretion.

\section{Concluding comments}

In conclusion, it is possible that airway gland secretions in CF are too concentrated and viscous to be cleared efficiently (3). Thus, anything that returns the viscosity of gland secretions to normal should alleviate the airway symptoms of CF. This hypothesis can now be tested in CFTR-deficient pigs and ferrets, species that (unlike the mouse) have substantial numbers of airway glands. The papers by Sun et al. (8) and Joo et al. (7) in this issue of the JCI are impor- tant in that they show that the CF-related reductions in gland fluid secretion in these animals qualitatively resemble those seen in human CF. Of the two species, pig airways and their mucous glands are the most similar to those of humans (Table 1 ). However, the usefulness of this species is limited by the $100 \%$ mortality in the perinatal period, due to gastrointestinal problems that can currently only be overcome surgically (6). In the ferret, by contrast, comparatively simple oral therapies can ensure survival of CFTR-deficient ferrets beyond the perinatal period (8). Thus, for now the ferret would seem the more useful model. The paper by Lee and Foskett (9) is a technical tour de force that provides the novel findings that cAMP elevates $\left[\mathrm{Ca}^{2+}\right]_{\mathrm{i}}$ in airway gland serous cells and potentiates the effects of carbachol on $\mathrm{Ca}^{2+}$ release (9). These findings point to pharmacological ways of enhancing (or inducing) baseline fluid secretion from CF glands without altering neurohumoral input.

Address correspondence to: Jonathan $\mathrm{H}$. Widdicombe, Department of Physiology and Membrane Biology, University of California Davis, Davis, California 95616-8664, USA. Phone: 530.754.7369; Fax: 530.752.5423; E-mail: jhwiddicombe@ucdavis.edu.

1. Widdicombe JH, Widdicombe JG. Regulation of human airway surface liquid. Respiration Physiology. 1995;99(1):3-12.

2. Reid L. Measurement of the bronchial mucous gland layer: A diagnostic yardstick in chronic bronchitis. Thorax. 1960;15:132-141.

3. Jayaraman S, Joo NS, Reitz B, Wine JJ, Verkman AS. Submucosal gland secretions in airways from cystic fibrosis patients have normal $\left[\mathrm{Na}^{+}\right]$and $\mathrm{pH}$ but elevated viscosity. Proc Natl Acad Sci U S A. 2001;98(14):8119-8123.

4. Guilbault C, Saeed Z, Downey GP, Radzioch D. Cystic fibrosis mouse models. Am J Respir Cell Mol Biol. 2007;36(1):1-7.

5. Sun X, et al. Adeno-associated virus-targeted disruption of the $\mathrm{cftr}$ gene in cloned ferrets. J Clin Invest. 2008;118(4):1578-1583.

6. Rogers CS, et al. Disruption of the cftr gene produces a model of cystic fibrosis in newborn pigs. Science. 2008;321(5897):1837-1841.

7. Joo NS, Cho H-J, Khansaheb M, Wine JJ. Hyposecretion of fluid from tracheal submucosal glands of CFTR-deficient pigs. J Clin Invest. 2010;120(9):3161-3166

8. Sun X, et al. Disease phenotype of a ferret CFTRknockout model of cystic fibrosis. J Clin Invest. 2010;120(9):3149-3160.

9. Lee RJ, Foskett JK. cAMP-activated $\mathrm{Ca}^{2+}$ signaling is required for CFTR-mediated serous cell fluid secretion in porcine and human airways. J Clin Invest. 2010;120(9):3137-3148.

10. Choi JY, et al. Synergistic airway gland mucus secretion in response to vasoactive intestinal peptide and carbachol is lost in cystic fibrosis. J Clin Invest. 2007;117(10):3118-3127.

11. Choi JY, et al. Substance P stimulates human airway submucosal gland secretion mainly via a cftr-depen- 
dent process. J Clin Invest. 2009;119(5):1189-1200.

12. Joo NS, Saenz Y, Krouse ME, Wine JJ. Mucus secretion from single submucosal glands of pig. Stimulation by carbachol and vasoactive intestinal peptide. J Biol Chem. 2002;277(31):28167-28175

13. Whimster WF. Number and mean volume of individual submucous glands in the human tracheobronchial tree. Appl Pathol. 1986;4(1-2):24-32.

14. Widdicombe JH, Pecson IS. Distribution and numbers of mucous glands in the horse trachea. Equine Vet J. 2002;34(6):630-633

15. Bedrossian CW, Greenberg SD, Singer DB, Hansen JJ, Rosenberg HS. The lung in cystic fibrosis. A quantitative study including prevalence of patho- logic findings among different age groups. Hum Pathol. 1976;7(2):195-204.

16. Tos M. Development of the tracheal glands in man. Number, density, structure, shape, and distribution of mucous glands elucidated by quantitative studies of whole mounts. Acta Pathol Microbiol Scand. 1966;68(suppl 185):3+

17. Leigh MW, Gambling TM, Carson JL, Collier AM, Wood RE, Boat TF. Postnatal development of tracheal surface epithelium and submucosal glands in the ferret. Exp Lung Res. 1986;10(2):153-169.

18. Salinas D, et al. Submucosal gland dysfunction as a primary defect in cystic fibrosis. FASEB $J$. 2005;19(3):431-433.
19. Cho H-J, Joo NS, Wine JJ. Mucus secretion from individual submucosal glands of the ferret trachea. Am J Physiol Lung Cell Mol Physiol. 2010; 299(1):L124-L136.

20. Joo NS, Wu JV, Krouse ME, Saenz Y, Wine JJ. Optical method for quantifying rates of mucus secretion from single submucosal glands. Am J Physiol Lung Cell Mol Physiol. 2001;281(2):L458-L468.

21. Choi HK, Finkbeiner WE, Widdicombe JH. A comparative study of mammalian tracheal mucous glands. J Anat. 2000;197 pt 3:361-372.

22. Robinson NP, Venning L, Kyle H. Quantitation of the secretory cells of the ferret tracheobronchial tree. J Anat. 1986;145:173-188.

\title{
Reconstructing neural circuits using transplanted neural stem cells in the injured spinal cord
}

\author{
Tamir Ben-Hur
}

Department of Neurology, The Agnes Ginges Center for Human Neurogenetics, Hadassah Hebrew University Medical School, Jerusalem, Israel.

\begin{abstract}
Traumatic spinal cord injury is one of the most common causes of disability in young adults. Restoring independent ambulation in such patients is considered one of the biggest challenges in regenerative medicine because repair of spinal cord injury involves the complex processes of axonal regeneration, remyelination, and formation of new synaptic connections. In this issue of the JCI, Abematsu et al. report their attempts to rise to this challenge, showing in a mouse model of severe spinal cord injury that spinal neuronal circuits can be restored by neural stem cell transplantation, leading to impressive functional recovery in the hind limbs.
\end{abstract}

Traumatic brain and spinal cord injury (SCI) is one of the most common causes of disability in young adults and poses a huge social and economical burden (1). The impact of nonpenetrating, blunt (contusive) trauma to the spinal cord causes abrupt discontinuation of axonal projections. There is also release of neurotoxic compounds and inflammatory mediators that add to neuronal and oligodendroglial cell death during the first hours and days after injury. This is followed by secondary processes of loss of myelin, degeneration of axons, and formation of a glial scar that inhibits spontaneous regeneration. Currently, there is no proven reparative treatment for SCI. Restoring independent ambulation in paraplegic trauma victims is considered one of the biggest challenges in regenerative medicine. This would require the development of approaches to achieve effective transmission of electrical impulses through the lesion. To this end, it is necessary to induce

Conflict of interest: The author has declared that no conflict of interest exists.

Citation for this article: J Clin Invest. 2010; 120(9):3096-3098. doi:10.1172/JCI43575. robust regeneration of severed axons, their covering with myelin sheaths - a process termed remyelination - and formation of new synaptic connections. With this notion in mind, studies of neural stem cell transplantation have been performed in experimental models of SCI during the last decade and have demonstrated increased growth of severed host axons and improved remyelination (2). In this issue of the JCI, Abematsu et al. (3) show that transplanting neural stem cells epigenetically directed to differentiate into neurons can promote the reconstruction of spinal neuronal circuits, leading to impressive functional recovery in mouse hind limbs following SCI.

\section{Impediments and therapeutic targets in spinal cord repair}

Restorative therapy in SCI should be directed against several targets. During the immediate hours and days after injury, the priority is to minimize inflammatory and neurotoxic damage. In the following days and weeks, the focus should be on halting the secondary processes of demyelination, axonal degeneration, and scar formation. However, meaningful recovery after SCI is ultimately an issue of axonal regeneration. There are several axonal projections passing through the spinal cord. The corticospinal tract consists of fibers that originate in cortical neurons and send axons extending through the brain and spinal cord (reaching over one meter long) to innervate spinal lower motor neurons. These, in turn, transmit the message for muscles to contract. Other descending tracts that are involved in execution of motor functions, such as the proprio-, rubro-, reticulo-, and raphespinal tracts, are multisynaptic and therefore consist of neurons with shorter axons. Direct growth of corticospinal fibers through the lesion to reinnervate distal segments that are caudal (below) to the site of injury is very limited, since corticospinal axons are the neuronal population with the least regenerative capacity. The multisynaptic spinal tracts are considered to have better inherent regenerative capacity than corticospinal tracts (4). Plastic mechanisms that have been shown to contribute to functional recovery after SCI include local sprouting of fibers to innervate undamaged collateral fibers, spinal interneurons, and multisynaptic tracts.

Restrictions to axonal regeneration in the CNS include both extrinsic and intrinsic factors (5). There are several known myelin- and extracellular matrix-derived molecules that are inhibitory to axonal regeneration. Removal of myelin (6), neutralization of myelin-derived inhibitory molecules such as Nogo (7), and neutralization of the extracellular matrix component chondroitin sulfate proteoglycan (8) all result in improved axonal growth. 